\title{
Successful Intensification of Intravenous Rheological Treatment of Phalanges Ischemia in SSc Patient Using Alprostadil
}

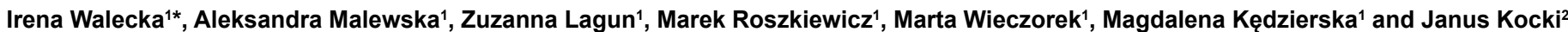

${ }^{1}$ Dermatology Department, Central Clinical Hospital of the Ministry of the Interior and Administration in Warsaw, Poland

${ }^{2}$ Department of Clinical Genetics, Medical University of Lublin, Poland

\begin{abstract}
Systemic sclerosis $(\mathrm{SSc})$ is a rare, auto immunological, connective tissue disease characterized by vasomotor disturbances, fibrosis and further atrophy of the skin and internal organs. There are many factors leading to immunological and vascular changes. One of the most prominent vascular symptoms is Raynaud's phenomenon, which may be caused by different factors such as cold or excessive stress. This case report presents a 37year old Caucasian woman with SSc, with severely impaired microcirculation of the hands and necrosis of the distal phalanges due to several hours of exposure to the cold. The patient required intensification of intravenous rheological treatment with alprostadil and oral usage of sildenafil, which had a positive effect on the improvement of the impaired microcirculation. Like many others, this case demonstrates that IV. rheological treatment may be effective in restoring microcirculation in such cases among SSc patients.
\end{abstract}

Keywords: Scleroderma; Systemic sclerosis; Impaired microcirculation; Alprostadil; Intravenous rheological treatment

\section{Introduction}

Systemic sclerosis (scleroderma, SSc) is an auto immunological, connective tissue disease characterized by vasomotor disturbances, fibrosis and further atrophy of the skin and internal organs [1].

There are many factors leading to immunological and vascular changes. Within the group of people with genetic predisposition, certain environmental factors damage the blood vessels and cause immune cell activation, which leads to non-specific stimulation of fibroblasts [2-4]. The excessive collagen deposition in tissues causes fibrosis of the skin and internal organs, being a characteristic feature of systemic sclerosis. Vascular changes, resulting from the involvement of vessels of various sizes, are a crucial factor causing the development of inflammation within the tissues in patients with systemic sclerosis. SSc is a rare condition, with a prevalence of around 20 per 100000 inhabitants and a female predominance overall. There are three main types of scleroderma: diffused scleroderma (dSSc), limited scleroderma (ISSc) and scleroderma without sclerosis (SSc sine sclerodema, SSSC) [5].

The presenting symptoms depend on the severity of organ involvement. The most common finding in patients with SSc is Raynaud's phenomenon, a sudden paling of distal parts of the body, followed by cyanosis and further occurrence of erythema in the last stage [6].

The involvement of the gastrointestinal tract is the most common, serious and prevalent complication of SSc, with the esophagus as the most frequently affected organ. The majority of patients suffer from difficulty in swallowing, nausea, vomiting, diarrhea or a fecal incontinence. The involvement of the gastrointestinal tract is a leading cause of morbidity in patients with systemic sclerosis [7].

Different types of SSc vary in the severity of skin sclerosis, process and prognosis of the disease, although the resulting changes to internal organs, bones and joints are the same. Diffused scleroderma is usually rapid and severe affecting the skin of the face, neck, trunk, upper and lower limbs. Raynaud's syndrome normally appears at the same time as sclerosis and telangiectasia are commonly seen. The face of dSSc patients becomes masklike with a thinning of the nose, lips, radial furrows, limited movability and microstomia (mouth narrowing). Callous skin edema is observed within the area of digital pulps. Ulcers within the ungual phalanges are a common finding and difficult to heal due to disturbances in the vasculature. Atrophic disturbance leads to bone destruction, followed by a shortening of the ungual phalanges (acroosteolysis).

Circumscribed scleroderma (ISSc) on the other hand, has a chronic and slow course. Typically, Raynaud's phenomenon is observed many years before the development of sclerosis. Skin changes affect the face and distal limb parts. Compared to dSSc, ISSc is characterized by less frequent pigmentation disturbances, cutis calcinosis around the joints of the hands and a greater increase in telangiectasia. Organ involvement is observed in late-stage 1SSc, whereas it is one of the first signs of in dSSc.

Vascular disorders have a crucial role in the pathogenesis of SSc. Erosions and ulcers, most commonly seen within the limbs, occur due to damaged blood vessels and chronic tissue ischemia. In many SSc patients, progressive structural changes developing within the minor arteries lead to permanent blood flow impairment and tissue necrosis [8].

\section{Case Report}

In our case review, we present a 37-year old Caucasian woman with SSc (positive anti-topoisomeraze I antibodies; diagnosed in July 2006) admitted to the Dermatology Department of the Central Clinical Hospital of the Ministry of the Interior and Administration in Warsaw with severely impaired microcirculation of the hands. At interview, her chief complaint was severe pain and paresthesia of the third finger of the right hand with skin pallor, which started 3 days prior to admission. 10 days earlier she had been exposed to cold for many hours and had subsequently suffered from flu-like symptoms.

*Corresponding author: Irena Walecka, Dermatology Department, Central Clinical Hospital of the Ministry of the Interior and Administration in Warsaw, Poland, Tel: 004805081813; E-mail: irena.walecka@cskmswia.pl

Received May 19, 2017; Accepted June 15, 2017; Published June 20, 2017

Citation: Walecka I, Malewska A, Lagun Z, Roszkiewicz M, Wieczorek M, et al. (2017) Successful Intensification of Intravenous Rheological Treatment of Phalanges Ischemia in SSc Patient Using Alprostadil. J Cardiovasc Dis Diagn 5: 284. doi: 10.4172/2329-9517.1000284

Copyright: ( 2017 Walecka I, et al. This is an open-access article distributed under the terms of the Creative Commons Attribution License, which permits unrestricted use, distribution, and reproduction in any medium, provided the original author and source are credited. 


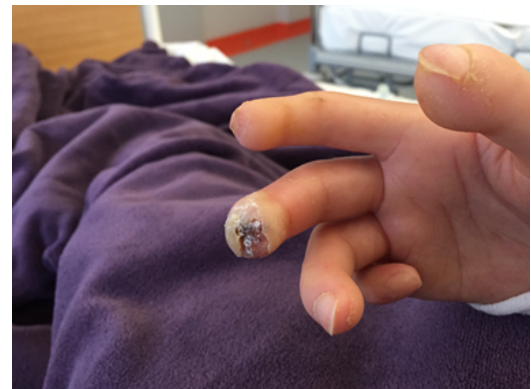

Figure 1: Ischemia area and necrosis of the third finger

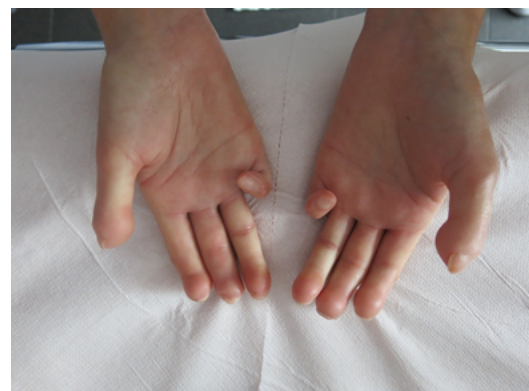

Figure 2: Condition after intense intravenous rheological treatment.

Upon general examination, the patient was found to be conscious and oriented; her vital signs were within the normal range. She presented with advanced Raynaud's phemonemon affecting both the fingers and toes. The third finger of the right hand was severely cyanotic with gray and blue discoloration due to chronic lack of tissue blood supply, which raised the suspicion of tissue necrosis (Figure 1).

The patient also presented a wide spectrum of classic scleroderma symptoms, such as advanced diffused skin fibrosis with hyper- and hypopigmentation, acroosteolysis resulting in a loss of distal phalanges, a claw-like appearance of the hands and acroosclerosis with painful ulcerations at the fingertips ('rat bite necrosis'). The loss of normal facial skin folds caused a mask-like appearance with concomitant radial perioral wrinkles, microstomy, thinning of the lips and diffused facial telenagiectasia. Moreover, the patient suffered from interstitial pulmonary fibrosis due to systemic sclerosis, hypothyroidism, recurrent erythema nodosum and latent tuberculosis infection. In the past, the patient had undergone different therapies for systemic sclerosis. She was treated with intravenous cyclophosphamide pulse therapy, methotrexate (use of this drug was stopped due to nausea), cyclosporine A (use of this drug was stopped due to gingival hyperplasia and mucous ulcerations) and prednisone [9-11]. Upon admission, the patient's therapy consisted of mycofenolate mofetil $2 \mathrm{~g}$ per day, sildenafil 12.5 mg per day, oral sulodexide 500 LSU per day and regular intravenous infusions of alprostadil every 8 -10 weeks $[12,13]$. Laboratory findings showed an accelerated sedimentation rate, hypokaliemia and hyperglicemia. A previous capillaroscopy of the fingers revealed a late phase of scleroderma pattern-an irregular enlargement of vascular loops, lack of hemorrhages and severe capillary atrophy of the avascular regions of the nail bed [6].

Due to the high risk of tissue necrosis, rheological treatment was intensified by multiple intravenous infusions of prostacycline analog-alprostadil ( $1 \mathrm{mcg} / \mathrm{kg}$ body weight- $60 \mathrm{mcg}$ per day for four days) and sulodexide along with standard doses of mycofenolate mofetil and analgesic therapy. After four days of such treatment, acral microcirculation was improved. However, due to continuous pain in the right hand, treatment with oxycodone

$20 \mathrm{mg}$ per day was continued. Before being discharged from the hospital, the patient's chronic therapy was also modified, the sildenafil dose was increased to $25 \mathrm{mg}$ per day (the highest tolerable dose), as well as the frequency of infusions of alprostadil.

Two weeks later the patient was admitted to the dermatology department for the second time, due to inflammation of the affected finger. An x-ray of the right hand showed osteolytic changes in the distal phalanges (previously observed), with no sign of bone inflammation. Doppler ultrasonography showed no flow in distal parts of the phalangeal arteries. A further alprostadil treatment was applied (60 mcg per day for five days) combined with antibiotic therapy (ciprofloxacine $800 \mathrm{mg}$ per day and metronidazole $1500 \mathrm{mg}$ per day).

The patient was discharged after the inflammation had been healed and microcirculation improved.

During the follow-up visits over the following 5 weeks, microcirculation was significantly improved with no signs of tissue necrosis, although no nail regrowth has been observed so far (Figure 2). Pain has weakened, allowing for a gradual reduction in oxycontine. The patient is now undergoing intensive therapy with alprostadil every 4-6 weeks.

\section{Conclusion}

There are multiple methods of treating patients with Raynaud's phenomenon and all of them share the same goal-the intensification of microcirculation in acral parts of the body. Apart from general measures (i.e. cessation of smoking, avoidance of cold temperatures and maintaining digital warmth) patients are treated with calcium channel blockers (CCBs), phosphodiesterase 5 inhibitors (sildenafil), al-selective alpha blocker (prazosin), selective serotonin reuptake inhibitor (SSRI) or topical nitrates. Also, regular administered intravenous rheological treatments (every 6-10 weeks) of prostacycline analogs are effective in improving impaired microcirculation in patients with SSc.

Recalcitrant Raynaud's phenomenon can be alleviated by administration of endothelin receptor antagonist - bosentan, which is commonly used in the treatment of pulmonary hypertension. Studies show, bosentan reduces the number of digital ulcers in scleroderma patients $[14,15]$.

There are also more invasive attempts to reduce vasospasm like digital sympatectomy or the use of botulinum toxin A (BTX A). The injection of BTX A into the perineurovascular tissue of the wrist or digit, blocks sympathetic nerve conduction and reduces vasospasm, thus significantly improves the healing process of digital ulcers $[16,17]$.

Similarly, digital sympatectomy can also improve microcirculation in the ischemic region. Raynaud's phenomenon can be reduced by various therapeutic options; however none of them is fully effective, especially in short period of time. In cases of acute phalangeal ischemia, like in presented patient, an intensive treatment is necessary. Therefore we believe that sequential therapy with two different intravenous rheological compounds (alprostadil and sulodexide) along with oral sildenafil is a promising, non-invasive method of avoiding amputation in patients with critical acute ischemia, who require quick and efficient treatment.

\section{References}

1. Park JS, Park MC, Song JJ, Park YB, Lee SK, et al. (2015) Application of the 
Citation: Walecka I, Malewska A, Lagun Z, Roszkiewicz M, Wieczorek M, et al. (2017) Successful Intensification of Intravenous Rheological Treatment of Phalanges Ischemia in SSc Patient Using Alprostadil. J Cardiovasc Dis Diagn 5: 284. doi: 10.4172/2329-9517.1000284

Page 3 of 3

2013 ACR/EULAR classification criteria for systemic sclerosis to patients with Raynaud's phenomenon. Arthritis Res Ther 17: 77

2. Marie I, Gehanno JF (2015) Environmental risk factors of systemic sclerosis Semin Immunopathol 37: 463-473.

3. Marie I, Gehanno JF, Bubenheim M, Duval-Modeste AB, Joly P, et al. (2014) Prospective study to evaluate the association between systemic sclerosis and occupational exposure and review of the literature. Autoimmun Rev 13: 151-156.

4. Marie I, Gehanno JF, Bubenheim M, Duval-Modeste AB, Joly P, et al. (2017) Systemic sclerosis and exposure to heavy metals: A case control study of 100 patients and 300 controls. Autoimmun Rev 17: 223-230.

5. Sakkas LI (2005) New developments in the pathogenesis of systemic sclerosis. Autoimmunity 38:113-116.

6. Walecka I, Sklodowska A, Roszkiewicz M, Wieczorek M, Lagun Z, et al. (2017) Raynaud's phenomenon: The clinical picture, treatment and diagnostics. Acta Angiol 23: 29-33.

7. Braun-Moscovici $Y$, Brun R., Braun M (2016) Systemic Sclerosis and the Gastrointestinal Tract: Clinical Approach. Rambam Maimonides Medical Journal 7: e 0031.

8. Szymanska E, Wieczorek M, Lagun Z, Malewska A, Roszkiewicz M, et al. (2016) Vascular changes in autoimmunological connective tissue diseases. Acta Angiol 22: 172-176.

9. Goldin J, Elashoff R, Kim HJ, Yan X, Lynch D, et al. (2009) Treatment of scleroderma-interstitial lung disease with cyclophosphamide is associated with less progressive fibrosis on serial thoracic high-resolution CT scan than placebo: Findings from the scleroderma lung study. Chest 136: 1333-1340.
10. Kowal-Bielecka O, Fransen J, Avouac J, Becker M, Kulak A, et al. (2015) Update of EULAR recommendations for the treatment of systemic sclerosis. Ann Rheum Dis 74: 90.3-91.

11. Omair MA, Alahmadi A, Johnson SR (2015) Safety and effectiveness of mycophenolate in systemic sclerosis. A systematic review. PLoS One 10: e0124205.

12. Hachulla E, Hatron PY, Carpentier P, Agard C, Chatelus E, et al. (2016) Efficacy of sildenafil on ischaemic digital ulcer healing in systemic sclerosis: the placebo-controlled SEDUCE study. Ann Rheum Dis 75: 1009-1015.

13. Tingey T, Shu J, Smuczek J, Pope J (2013) Meta-analysis of healing and prevention of digital ulcers in systemic sclerosis. Arthritis Care Res 65 1460-1471.

14. Korn, JH, Mayes M, Matucci-Cerinic M, Rainisio M, Pope J, et al. (2004) Digital ulcers in systemic sclerosis: Prevention by treatment with bosentan, an oral endothelin receptor antagonist. Arthritis and Rheumatism 50: 3985-3993.

15. Matucci-Cerinic M, Denton CP, Furst DE, Mayes MD, Hsu VM, et al. (2010) Bosentan treatment of digital ulcers related to systemic sclerosis: Results from the RAPIDS-2 randomised, double-blind, placebo-controlled trial. Ann Rheum Dis 70: $32-38$.

16. Fregene A, Ditmars D, Siddiqui A (2009) Botulinum Toxin Type A: A Treatment option for digital ischemia in patients with raynaud's phenomenon. Journal of Hand Surgery 34: 446-452.

17. Uppal L, Dhaliwal K, Butler PE (2013) A prospective study of the use of botulinum toxin injections in the treatment of Raynaud's syndrome associated with scleroderma. Journal of Hand Surgery 39: 87-880. 